\title{
The use of bitter herbs in practice
}

\begin{abstract}
The use of bitter herbs in treatment of digestive disorders is common to many traditional healing systems. This article will reason that improvements of wellbeing after ingesting bitters are due to reflex physiological responses elicited by oral receptors. Any improvement is certainly not a placebo effect even if science has had been slow in determining the bitter tastants mechanism of activity. The article gives an update on oropharyngeal taste physiology, an overview of the digestive process emphasizing postprandial hyperemia, an evidence based critical review of the suggested mechanisms by which bitters support digestion and suggestions as to when and why bitters can be applied for digestive and cardiovascular problems. Through understanding the mechanism by which traditional medicines work, we can both substantiate traditional usage and refine our tools. This knowledge allows us to extend traditional preparations to other pathologies. Our traditional knowledge base is large, but to investigate it we need experimental models sensitive enough to detect physiological changes that do not involve agents that block physiological activity. The studies presented in this article give only a glimpse of the effect bitters have on the body. Much is still to be discovered within the chemosensory world of tastants.
\end{abstract}

Volume 6 Issue 5 - 2017

\author{
Michael McMullen \\ Independent Researcher, France
}

Correspondence: Michael McMullen, Independent Researcher, France, 53 Rue Blanquerie, I | 300 Limoux, France, Tel +33 688 032938,Email research@micmcmullen.se

Received: January 25, 2017 | Published: April 25, 2017

Keywords: artemisia absinthium, gentiana lutea, bitter tastant, cephalic phase responses, gastroparesis, postprandial hyperemia, postprandial hypotension, taste receptors cell, total peripheral resistance, vascular peripheral resistance

Abbreviations: TRC, taste receptors cell; hTAS2R, human bitter taste receptor; GPRC, g protein coupled receptors; $\mathrm{dP} / \mathrm{dt}$, cardiac contraction force

The use of bitter herbs in treatment of digestive disorders is common to many traditional healing systems. This article will reason that an improvement of wellbeing after ingesting bitters is due to reflex physiological responses elicited by oral receptors. Any improvement is certainly not a placebo effect even if science has had been slow in determining the bitter tastant mechanism of activity. ${ }^{1,2}$ This article will provide

i. An update on oropharyngeal taste physiology,

ii. an overview of the digestive process emphasizing postprandial hyperemia,

iii. an evidence based critical review of the suggested mechanisms by which bitters support digestion, and

iv. Suggestions as to when and why bitters can be applied for digestive and cardiovascular problems.

\section{Oropharyngeal taste physiology}

Tastes buds containing taste receptors cells (TRC) are located in the oropharyngeal cavity to provide information about ingested material. The molecular structures of the oropharyngeal TRCs have been identified: G protein coupled receptors (GPRC) for the bitter, sweet and umami tastes and ion channels for the salty and sour tastes. The human genome encodes more than 30 GPRCs and 25 of these are involved with the bitter taste. ${ }^{3}$

Reports indicate that bitter compounds, found in some of the best known bitter remedies stimulate multiple bitter receptors (hTAS2Rs). For example, absinthin stimulates the hTAS2Rs 10, 14, 46 and 47, amarogentin stimulates the hTAS2Rs 1, 4, 39, 43, 46, 47 and 50, Andrographolide stimulates the hTAS2Rs 46,47 and 50, caffeine stimulates the hTAS2Rs 7, 10, 14, 43 and 46, humulone isomers stimulates the hTAS2Rs 1,13 and 40 , picrotoxinin stimulates the
hTAS2Rs 1, 10, 14, 46 and 47, quassin stimulates the hTAS2Rs 4, 10, 14,46 and 47 , quinine stimulates the hTAS2Rs $4,7,10,14,39,40$, 43,44 , and $46 .{ }^{4}$

This information is important to therapists because it presents the concept that different bitters may elicit distinct neural signaling and elicit distinct reflex responses. However, it is widely accepted that there is only one representation of bitterness at the level of awareness so does the hTAS2R signaling merge at the cellular level to produce one bitter neuron or do 25 separate bitter neurons emerge from the TRC and merge somewhere in the brain? These two alternatives are referred to as the across-fiber pattern model and the label-lined model. ${ }^{5}$

\section{The digestive process}

Digestion is a process that takes over time and involves the secretion of saliva, swallowing, movement of the bolus along the esophagus, gastric distension, gastric secretions, gastric emptying, pancreatic secretions, gallbladder contractions, small intestine secretions, small intestine movement, absorption of nutrients, etc. These functions all require blood to supply nutrients to the metabolically active cells and blood to remove metabolic wastes as well as to remove the absorbed nutrients. Consequently, the splanchnic blood flow is a major limiting factor for normal digestion.

Digestion involves three distinct phases. The initial phase, the cephalic phase, involves thought, anticipation, sight, smell, taste, mouth sensations and swallowing. ${ }^{6}$ Nevertheless, the extent of cephalic phase responses after decreases in the following order: swallowing $>$ mastication $>$ taste $>$ smell $>$ sight $>$ thought. ${ }^{7}$ Increases of flow in the celiac artery begin during the cephalic phase and continue for approximately 10 to 15 minutes after ingestion, a period referred to as the gastric phase. ${ }^{8}$ During the gastric phase the ingested material triggers tension sensitive mechanoreceptors which stimulate gastric relaxation and stomach secretions via intrinsic and vago-vagal pathways. It is thought that there is minimal chemosensory activity in the stomach. ${ }^{9}$ When gastric emptying begins, the flow in the celiac 
artery returns to pre-ingestion levels, the gastric phase ends and the intestinal phase starts. The intestinal phase is characterized by an increased blood flow in the superior mesenteric artery ${ }^{8}$ and can last for more than four hours. ${ }^{10}$ Blood flow may increase from 800 to $1000 \mathrm{~mL} /$ minute in the celiac artery during the gastric phase and from 500 to $1400 \mathrm{~mL} /$ minute in the superior mesenteric artery during the intestinal phase. ${ }^{11}$

The circulatory response to the ingestion of foods and drink, known as postprandial hyperemia, has been extensively studied in both humans ${ }^{12,13}$ and $\operatorname{dog} \mathrm{s}^{14}$ during the 1980s and 1990s. Researchers reported that, for healthy individuals, postprandially both heart rate and cardiac output increased, blood pressure was unchanged while vascular peripheral resistance decreased. ${ }^{13}$ The magnitude of the cardiovascular effects was directly related to meal size, with only the largest meal affected calf blood flow. Furthermore, It was reported that, while a high carbohydrate meal and a high fat meal elicited similar cardiac changes, the fat meal had a more prolonged effect on calf blood flow than did the carbohydrate meal. ${ }^{12}$

Until recently it was assumed that the only postprandial cardiac response is an increase in heart rate, however contraction force $(\mathrm{dP} / \mathrm{dt})$ can also increase blood pressure postprandially. ${ }^{15}$ The increased dP/ $\mathrm{dt}$ is of such intensity that resultant elevations of blood pressure lead to decreases of heart rate even as cardiac output remains unchanged. In cardiovascular studies, $\mathrm{dP} / \mathrm{dt}$ is frequently overlooked parameter. ${ }^{16}$

An inadequate postprandial hemodynamic response, due either to cardiac insufficiency or a drop in vascular peripheral resistance as the circulatory volume increases due to extra flow to the splanchnic circulation can be disastrous for the body resulting in both digestion and circulatory dysfunction. Postprandial hypotension is defined as a drop in systolic pressure of $\geq 20 \mathrm{mmHg}$, or a decrease below $90 \mathrm{mmHg}$, within 2 hours of eating. Postprandial hypotension can result in falls, syncope, coronary events and strokes ${ }^{17}$ and it is associated with both coronary events and overall mortality ${ }^{18,19}$

\section{Bitter tastant theories}

\section{The "vagal-stimulation" or "cephalic response" theory}

"Bitter stimuli pass primarily by way of the glossopharyngeal nerve to a special group of cells in the cerebral cortex. The taste is interpreted there as bitter, and causes stimuli to be forwarded through the vagus nerve to both the salivary gland and the stomach .... This stimulation of the digestive processes enhances the appetite". ${ }^{20}$ This is the most popular theory amongst writers of herbal texts and probably nearly all herbal practitioners would support it. It is endorsed in the following influential literatures Natural Remedies. Their Origins and uses, ${ }^{21}$ rational Phytotherapy. A Reference Guide for Physicians and Pharmacists, ${ }^{22}$ Principles and Practice of Phytotherapy. Modern Herbal Medicine $^{23}$ and the European Medical Agency (EMA) Assessments of Artemisia absinthium $\mathrm{L}^{24}$ and Gentian lutea L. ${ }^{25}$

One group of pharmacologists ${ }^{22}$ citing earlier work ${ }^{26}$ proposed that "bitter principles act reflexively on the cardiovascular system causing a decrease of in heart rate and cardiac stroke volume" i.e. increase vagal tone However, the statistical analysis upon which this concept is based has been shown to be faulty, and there is no evidence from cardiovascular research indicating that bitter tastants increase vagal tone. ${ }^{27}$

Despite the strong belief of many authors in this mechanism, after an extensive review the EMA assessment concluded for Gentian lutea "it is well-known that the bitter constituents stimulate the gustatory nerves in mouth and possibly increase the secretion of gastric fluid and bile, thereby enhancing appetite and digestion, while the detailed molecular mechanism of such activities is still to be investigated". ${ }^{25}$ This means that despite a widespread belief in this mechanism, there is insufficient evidence to support the "vagalstimulation" or "cephalic response" theory. Yet, it should also be noted that while It is well accepted that cephalic phase responses influence digestion ${ }^{28}$ tools to measure digestive secretions are not routinely available for researchers. ${ }^{29}$

\section{Local-response}

Bitter tastants "act directly on the mucosa of the upper part of the gastrointestinal tract and especially on the bitter receptors of the tongue stimulating the release of saliva and gastric juices". ${ }^{2}$ This view is favored by non-practitioners and pharmacologists, but is perhaps nothing more than a non-vagal proposal to explain the observed activity of bitters. This view does receive support from the observation that taste receptors are located throughout the rodent gut, ${ }^{30}$ referred to as the diffuse chemosensory system. ${ }^{31}$ However, it is widely believed that there are few sensory cells located in the stomach wall ${ }^{9}$ and any sensory cells beyond the stomach are unlikely to be active in the gastric phase of digestion. Some proponents of the vagal theory accept that local stimulation may adjunct cephalic responses ${ }^{20,25}$ others state that if bitter tastants are to be effective "they must be tasted". ${ }^{23}$

\section{Circulatory support}

Over a 100years ago in the book Materia Medica, Pharmacy, Pharmacology and Therapeutics, it was proposed that the mechanism by which bitters influence digestion was as follows: "the appetite is sharpened because the gustatory nerves are stimulated, this reflexively leads to dilation of the gastric vessels and to an increase in the gastric and salivary secretions". ${ }^{32}$ Similarly, Weiss proposed that "the appetite-inducing action of bitters is probably due to improved circulation in the abdominal organs" ${ }^{\prime 33}$ Weiss also referred to action of bitters as sympathetic stimulation and suggested that the tonic action of bitters was the result of repeated sympathetic stimulation of the nervous system.

\section{Evidence}

My research group carried out a series of studies to test the above theories. In the first two we examined the effect of regular arabica coffee (containing circa 130mg caffeine), decaffeinated arabica coffee and caffeine $(133 \mathrm{mg})$ capsules..$^{34,35}$ Decaffeinated coffee, containing quinides, which are responsible for coffees bitter taste, ${ }^{36}$ had no effect on cardiovascular parameters whereas regular coffee, containing both caffeine and quinides, increased heart rate for at least 30minutes. These studies reported that caffeine stimulates oropharyngeal hTAS2Rs, eliciting a sympathetic response, while the quinides, which produce the same hedonic bitter response, elicited no cardiovascular response. Caffeine capsules after opening in gut elicited a decrease in arterial compliance and an increase diastolic pressure, 10-15minutes post-ingestion, but only during the time it was present in the stomach $(<15$ mins). This confirms the presence of hTAS2R receptors in the stomach.

In the next study, we tested the impact of fluid extracts (500 and $1500 \mathrm{mg}$ ) and encapsulated (1000mg) preparations of Artemisia absinthium and Gentiana lutea. In contrast to the caffeine capsules, the encapsulated bitters elicited no cardiovascular response. The differing responses produced by bitters demonstrates again that there is no generalized bitter effect in the gut and emphases that just because hTAS2Rs are present in the gut we cannot infer that all 25 types are present or that they elicit a systemic response. On the other hand, 
the bitter fluid preparations elicited increases in vascular peripheral resistance demonstrating that two bitters, well-known for their support of the digestive processes, stimulate oropharyngeal hTAS2Rs eliciting reflex autonomic responses that alter postprandial hemodynamic. Furthermore, the increases of vascular peripheral resistance resulted in a reduction of the cardiac workload. Thus, the response elicited by the taste of Artemisia absinthium and Gentiana lutea can support the postprandial hyperemia by increasing vascular tonus and reduce demands on the heart. In the statistical analysis, differences were obtained between the pre- and post-ingestion 5-10minutes (placebo controlled). Later, we also compared the between the preand post-ingestion 25-30minutes (placebo controlled) and found no difference. This does not necessary mean that the bitter effect is short-lived because after circa 30minutes in the upright posture, there cardiovascular accommodations that increase peripheral vascular resistance and act as a confounder. ${ }^{37}$

\section{The application of bitters digestive and cardiovascular problems}

One of the key findings of the above studies is that there is no generalized bitter effect, so the hedonics of taste do not indicate potential reflex responses. This indicates that at some point in the neural messaging from the mouth to the awareness centers, the neural transmissions from the different TAS2Rs must converge. This converge point/s does not lie in the oropharyngeal cavity as different bitter tastants elicit different cardiovascular responses. Consequently, neural transmission from the oropharyngeal bitter receptors to the medulla is partially or completely labelled-lined. ${ }^{38}$

\section{Types of usage}

The use of bitter herbs which act to support digestion is likely to be limited to situations where postprandial hyperemia is inadequate.

Acute situations: E.g. overindulgence as occurs with parties and feasts. The overindulge temporary overwhelms the cardiovascular capacity and does not reflect the normal capacity to adequately to digest meals. Symptoms may include bloating, nausea, and gastric reflux or even vomiting, heart palpitations, heart attack, syncope, stroke, night sweats and headaches. The symptoms experienced occasionally may be more intensive than those of chronic sufferers who tend to limit meal size intake to avoid discomfort. Thus, most people, even children, with normal cardiovascular capacity will benefit from these bitters if they have acute digestive problems.

Pathological chronic situations: Pathological chronic situations such as dyspepsia, heart conditions, diabetes, postural hypotension and postprandial hypotension may benefit from the use of bitters increasing peripheral vascular resistance. As in all treatments of pathologies, individual responses may vary widely and increases in peripheral vascular resistance are dependent on an intact sympathetic nerve transmission to the circulatory resistance vessels. This treatment may provide a useful adjunct to other treatments with the herbs having the advantage of low toxicity. Although Artemisia absinthium has a reputation for toxicity this opinion does not hold up to scrutiny ${ }^{39}$ and a $45 \%$ alcohol percolation extract of this plant does not contain measurable levels of essential oils, such as thujone, ${ }^{15}$ a result consistent with other research. ${ }^{40}$

Gastroparesis: Gastroparesis is a condition where there is a partial paralysis of the stomach muscles. This results in delayed gastric emptying and causes food to remain in the stomach. Symptoms include boating, nausea, vomiting, heart palpitations, poor appetite, gastric reflux, weight loss, night sweats and headaches. Gastroparesis is a problem for longstanding diabetic patients, as well as much other pathology. An open study using a traditional Japanese bitter tasting herbal compound, Rikkunshi, indicated that it enhanced gastric emptying for a group of medicated Parkinson's patients diagnosed with gastroparesis. ${ }^{41}$ The study was conducted over 12 weeks and the participant's mean age was 70years. Symptom improvement included improved appetite and reduced bloating. Similarly, a systemic review of the effects of the Chinese herbal compound, Xiangshaliujunzi Decoction, reported improved gastric emptying and reduced symptoms following treatments for 2 to 8 weeks. ${ }^{42}$ Both these combinations contain similar plants, including the bitter tasting Atractylodis species. Whether these preparations acted directly in the stomach or by increasing vascular peripheral resistance and facilitating gastric emptying is difficult to differentiate, however gastric emptying is dependent on adequate postprandial hyperemia.

Postprandial hypotension: Elderly people often lose their appetite and the initiate behavior patterns that justify this situation. In elderly homes, we hear comments that "the meals are uninteresting", "the meals are too large", "I don't like that", "today I am not hungry", "the food is tasteless" etc. while at the same time the elderly are losing weight and getting shorter. I suggest that many elderly have a comprised postprandial hyperemia due to a failing cardiovascular system which is causing them to slowly starve. Postprandial hypotension was first described only in $1977^{43}$ and it is difficult to detect clinically unless blood pressure measurements are taken after meals. The prevalence in institutionalized elders has been estimated at $25 \%-38 \%{ }^{17}$ although a study in Holland reported a prevalence of $67 \%$ amongst geriatric patients. ${ }^{44}$ One study concluded that "in older low-level-care residents, postprandial hypotension is an independent predictor of all-cause mortality with no added predictive value explained by other blood pressure indices: orthostatic hypotension, hypertension, pulse pressure or mean arterial pressure". ${ }^{18}$ As Artemisia absinthium and Gentiana lutea increase vascular peripheral resistance, which acts to increase blood pressure and reduce cardiac workload they have the potential to reduce the burden of postprandial hypotension.

Tonic activity: the use of bitter herbs is associated with a tonic effect ${ }^{33}$ i.e., an gradual and permanent increase in vitality and wellbeing over time. This effect can be explained on the basis that by increasing the digestive capacity, both the appetite and nutrition status can improve. But there are other areas that may also be affected. If the digestive system organs have been suffering from relative ischemia over many years, the increase in blood flow provided by improved postprandial hyperemia can remove accumulated metabolic wastes and improve organ function. Also, by improving postprandial hyperemia by increasing vascular peripheral resistance, the requirements on the heart are reduced and there is a greater blood flow to the brain and all other organs during the postprandial period.

\section{Dosage}

The dosages studied were $100 \mathrm{~mL}$ water flavored with $500 \mathrm{mg}$ and $1500 \mathrm{mg}$ in fluid extract form with the higher dose producing the larger effect. These dosages are consistent with European traditions ${ }^{24,25,32}$ but much larger than doses recommended by some modern authors. ${ }^{23}$ During our study, participants quickly swallowed the bitter flavored water so that exposure to hTAS2Rs was minimal. Despite this limited exposure time some participants spontaneously reported that the bitter taste was present 30 minutes later. Obviously, any potential alternative preparation must be tasted so lozenges, chewing gum or mouth sprays may be a viable and cheaper proposition. Oddly humans like bitter drinks, such as beer, coffee and tea, yet are much more restrictive with bitter foods. This suggests mouth sprays may be preferable to 
lozenges or chewing gum. It is likely that just as humans learn to drink bitter tasting coffee by experiencing it's mentally stimulating effects ${ }^{45}$ during or soon after drinking, we could expect that humans receiving digestive benefits soon after exposure to either Artemisia absinthium or Gentiana lutea would quickly learn the positive aspects of the intake of the bitter tastant. It has yet to be determined how repeated dosing would affect vascular peripheral resistance. One thing is certain though and that is that the hTAS2Rs do not become quickly exhausted otherwise the second cup of coffee or glass of beer would taste like water. Traditionally Gentiana lutea based aperitifs, with alcohol content circa 20\%, are common in southern France. My local supermarket sells at least five different types which seem to be most popular with the elderly. The greatest effect is likely to be when drunk with ice, but that is another physiology!

\section{Conclusion}

Through understanding the mechanism by which traditional medicines work, we can both substantiate traditional usage and refine our tools. We can also extend traditional preparations to other pathologies. Our traditional knowledge base is large, but to investigate it we need experimental models sensitive enough to detect physiological changes that do not involve agents that block physiological activity. The studies above are only a glimpse of the effect bitters have on the body. Much is still to be discovered within the chemosensory world of tastants.

\section{Acknowledgments}

None.

\section{Conflicts of interest}

Author declares there are no conflicts of interest.

\section{Funding}

None.

\section{References}

1. Laurence DR, Bennett PN, Brown MJ. Clinical Pharmacology. 8th edn. New York, USA: Churchill Livingstone; 1997. pp. 1-10.

2. Heinrich MJ, Barnes S, Gibbons S, et al. Fundamentals of Pharmacognosy and Phytotherapy. 2nd edn. Edinburgh, UK: Churchill Livingstone; 2012. pp. 1-326.

3. Meyerhof W, Born S, Brockhoff A, et al. Molecular Biology of Mammalian Bitter Taste Receptors. A Review. lavour Frag $J$. 2011;26(4):260-268.

4. Meyerhof W, Batram C, Kuhn C, et al. The Molecular Receptive Ranges of Human Tas2r Bitter Taste Receptors. Chem Senses. 2010;35(2):157170.

5. Carleton A, Accolla R, Simon SA. Coding in the Mammalian Gustatory System. Trends Neurosci. 2010;33(7):326-334.

6. Smeets PA1, Erkner A, de Graaf C. Cephalic Phase Responses and Appetite. Nutr Rev. 2010;68(11):643-655.

7. Mattes RD. Physiologic Responses to Sensory Stimulation by Food: Nutritional Implications. J Am Diet Assoc. 1997;97(4):406-413.

8. Someya N, Endo MY, Fukuba Y, et al. Blood Flow Responses in Celiac and Superior Mesenteric Arteries in the Initial Phase of Digestion. Am J Physiol-Regul Integr Comp Physiol. 2008;294(6):R1790-R1796.

9. Farré R, Tack J. Food and Symptom Generation in Functional Gastrointestinal Disorders: Physiological Aspects. Am J Gastroenterol. 2013;108(5):698-706.
10. Heinrich H, Goetze O, Menne D, et al. Effect on Gastric Function and Symptoms of Drinking Wine, Black Tea, or Schnapps with a Swiss Cheese Fondue: Randomised Controlled Crossover Trial. BMJ. 2010;341:c6731.

11. Mensink PB, Moons LM, Kuipers EJ. Chronic Gastrointestinal Ischaemia: Shifting Paradigms. Gut. 2011;60(5):722-737.

12. Sidery MB, Macdonald IA, Cowley AJ, et al. Cardiovascular Responses to High-Fat and High-Carbohydrate Meals in Young Subjects. Am J Physiol. 1991;261(5 Pt 2):H1430-H1436.

13. Sidery MB, Macdonald IA. The Effect of Meal Size on the Cardiovascular Responses to Food Ingestion. Br J Nutr. 1994;71(6):835-848.

14. Chou CC, Coatney RW. Nutrient-Induced Changes in Intestinal BloodFlow in the Dog. Br Vet J. 1994; (5):423-437.

15. McMullen MK, Whitehouse JM, Whitton P, et al. Bitter Tastants Alter Gastric-Phase Postprandial Haemodynamics. J Ethnopharmacol. 2014;154(3):719-727.

16. McMullen MK. Oops! Who Forgot to Measure Cardiac Contraction Force (Dp/Dt)? Austin J Pharm Ther. 2014;11(4):1-4.

17. Luciano GL, Brennan MJ, Rothberg MB. Postprandial Hypotension. Am J Med. 2010;123(3):281.e281-e286.

18. Fisher AA, Davis MW, Srikusalanukul W, et al. Postprandial Hypotension Predicts All-Cause Mortality in Older, Low-Level Care Residents. J Am Geriatr Soc. 2005;53(8):1313-1320.

19. Trahair LG, Horowitz M, Jones KL. Postprandial Hypotension: A Systematic Review. J Am Med Dir Assoc. 2014;15(6):394-409.

20. Robbers JE, Tyler VE. Tyler's Herbs of Choice. Binghamton, USA: The Hawthorn Press; 1999. Pp. 1-187.

21. Sandberg F, Corrigan D. Natural Remedies. Their Origins and Uses. London, UK: Taylor and Thomas; 2001. pp. 1-169.

22. Schulz VR, Hänsel, Tyler VE. Rational Phytotherapy. A Reference Guide for Physicians and Pharmacists. 5th edn. Berlin, Germany: Springer; 2004. p. 1-417.

23. Mills S, Bone K. Principles and Practice of Phytotherapy. Modern Herbal Medicine. (2nd edn), Churchill Livingstone, Edinburg, pp. 2013;1-1056.

24. Koch J. Assessment Report on Artemisia Absinthium L., Herba. European Medicines Agency, London, p. 2009;1-26.

25. Knöss W, Stolte F. Assessment Report on Gentiana Lutea L., Radix. London, UK: European Medicines Agency; 2009. p. 1-19

26. Glatzel H. Influence of Bitter Tonics on Heart Stroke Volume, Heart Beat Frequenccy and Blood Pressure. Planta Med. 1968;16(1):82-94.

27. McMullen MK. Evidence Does Not Support the Suggestion That Gustatory Exposure to Bitter Tastants Reduces Cardiac Activity. $J$ Ethnopharmaco. 2013;149(3):838-839.

28. Zafra MA, Molina F, Puerto A. The Neural/Cephalic Phase Reflexes in the Physiology of Nutrition. Neurosci Biobehav Rev. 2006;30(7):10321044.

29. Furness JB. The Enteric Nervous System. Malden: Blackwell; 2006. pp. $1-288$.

30. Horn CC. Electrophysiology of Vagal Afferents. Ann N Y Acad Sci. 2009;1170(1):69-76.

31. Sbarbati A, Bramanti P, Benati D, et al. The Diffuse Chemosensory System: Exploring the Iceberg toward the Definition of Functional Roles. Prog Neurobiol. 2010;91(1):77-89.

32. Hale White W. Materia Medica, Pharmacy, Pharmacology and Therapeutics. 1st edn. London, UK: $J \&$ A. Churchill; 1892. pp. 1-614. 
33. Weiss RF. Herbal Medicine. 6th edn. Sweden: AB Arcanum, Gothenburg, 1988. pp. 1-384.

34. McMullen MK, Whitehouse JM, Shine G, et al. The Immediate and Short-Term Chemosensory Impacts of Coffee and Caffeine on Cardiovascular Activity. Food Funct. 2011;2(9):547-554.

35. McMullen MK, Whitehouse JM, Shine G, et al. Caffeine in Hot Drinks Elicits Cephalic Phase Responses Involving Cardiac Activity. Food Funct. 2012;3(9):931-940.

36. Frank O, Zehentbauer G, Hofmann T. Bioresponse-Guided Decomposition of Roast Coffee Beverage and Identification of Key Bitter Taste Compounds. Eur Food Res Technol. 2006;222(5-6):492508.

37. Ricci F, De Caterina R, Fedorowski A. Orthostatic Hypotension: Epidemiology, Prognosis, and Treatment. $J$ Am Coll Cardiol. 2015;66(7):848-860.

38. McMullen MK.Neural Transmission from Oropharyngeal Bitter Receptors to the Medulla Is Partially or Completely Labelled-Line. Nat Prod Commun. 2016;11(8):1201-1204.

39. Padosch SA, Lachenmeier DW, Kröner LU. Absinthism: A Fictitious 19th Century Syndrome with Present Impact. Subst Abuse Treat Prev Policy. 2006;1(1):1-14
40. Tegtmeier M, Harnischfeger G. Methods for the Reduction of Thujone Content in Pharmaceutical Preparations of Artemisia, Salvia and Thuja. Eur J Pharm Biopharm. 1994;40(5):337-340.

41. Doi H, Sakakibara R, Sato M, et al. Dietary Herb Extract Rikkunshi-to Ameliorates Gastroparesis in Parkinson's Disease: A Pilot Study. Eur Neurol. 2014;71(3-4):193-195.

42. Tian JX, Li, Liao JQ, et al. Xiangshaliujunzi Decoction for the Treatment of Diabetic Gastroparesis: A Systematic Review. World J Gastroenterol. 2014;20(2):561-568.

43. Seyer-Hansen K . Postprandial Hypotension. $\mathrm{Br}$ Med J. 1977;2(6097):1262.

44. Vloet LC, Pel-Little RE, Jansen PA, et al. High Prevalence of Postprandial and Orthostatic Hypotension among Geriatric Patients Admitted to Dutch Hospitals. J Gerontol Ser a-Biol Sci Med Sci. 2005;60(10):1271-1277.

45. Yarmolinsky DA, Zuker CS, Ryba NJ. Common Sense about Taste: From Mammals to Insects. Cell. 2009;139(2):234-244. 